\begin{tabular}{|c|c|}
\hline Title & Percutaneous insertion of hepatic fiducial true spherical markers for real-time adaptive radiotherapy \\
\hline Author(s) & $\begin{array}{l}\text { Morita, Ryo; A bo, Daisuke; Sakuhara, Yusuke; Soyama, Takeshi; Katoh, Norio; Miyamoto, Naoki; Uchinami, } \\
\text { Yuusuke; Shimizu, Shinichi; Shirato, Hiroki; Kudo, Kohsuke }\end{array}$ \\
\hline Citation & $\begin{array}{l}\text { Minimally invasive therapy and allied technologies, 29(6), 334 } 343 \\
\text { https://doi.org/10.1080/13645706.2019.1663217 }\end{array}$ \\
\hline Issue Date & 2019-09-13 \\
\hline Doc URL & http:/hdl .handle.net/2115/79242 \\
\hline Rights & $\begin{array}{l}\text { This is an A ccepted Manuscript of an article published by Taylor \& Francis in Minimally Invasive Therapy \& A llied } \\
\text { Technologies on } 13 \text { Sep 2019, available online: http:/www.tandfonline.com/10.1080/13645706.2019.1663217. }\end{array}$ \\
\hline Type & article (author version) \\
\hline File Information & MITAT_20190913.pdf \\
\hline
\end{tabular}

Instructions for use 


\section{Percutaneous insertion of hepatic fiducial true-spherical markers for}

\section{2 real-time adaptive radiotherapy}

3 Ryo Morita ${ }^{\mathrm{a}}$, Daisuke Abo ${ }^{\mathrm{a} *}$, Yusuke Sakuhara ${ }^{\mathrm{b}}$, Takeshi Soyama ${ }^{\mathrm{a}}$, Norio

4 Katoh $^{\mathrm{c}, \mathrm{d}}$, Naoki Miyamoto ${ }^{\mathrm{c}}$, Yuusuke Uchinami ${ }^{\mathrm{c}}$, Shinichi Shimizu ${ }^{\mathrm{d}, \mathrm{e}}$,

$5 \quad$ Hiroki Shirato ${ }^{\mathrm{d}, \mathrm{f}}$, Kohsuke Kudo ${ }^{\mathrm{a}, \mathrm{d}}$

6 a Department of Diagnostic and Interventional Radiology, Hokkaido University

7 Hospital, Sapporo, Japan

$8 \quad{ }^{b}$ Department of Diagnostic Radiology, Tonan Hospital, Sapporo, Japan

9 'Department of Radiation Oncology, Hokkaido University Hospital, Sapporo, Japan

$10{ }^{d}$ Global Station for Quantum Medical Science and Engineering, Global Institution for

11 Collaborative Research and Education (GI-CoRE), Hokkaido University, Sapporo,

12 Japan

13 e Department of Radiation Oncology, Hokkaido University Faculty of Medicine,

14 Sapporo, Japan

15 fProton Beam Therapy Center, Hokkaido University Hospital, Sapporo, Japan

$16 *$ Corresponding author 
17 Daisuke Abo

18 Department of Diagnostic and Interventional Radiology, Hokkaido University Hospital

19 N-14, W-5, Kita-ku, Sapporo, Hokkaido 060-0848, Japan

20 Phone: $+81-11-716-1161$

21 Fax: +81-11-706-7876

22 E-mail: abodai@med.hokudai.ac.jp

23 Word count: 3264

24 Meeting presentations: This work was presented at RSNA 103rd Scientific Assembly and Annual

25 Meeting, Chicago, 2017.

26 Funding: This study was not supported by any funding.

27 Disclosures: Yuusuke Uchinami declares no conflict of interest. Ryo Morita received grants from the

28 Japan Agency for Medical Research and Development (AMED), unrelated to this study. Daisuke Abo

29 received grants from Siemens Medical Systems and AMED, unrelated to this study. Yusuke Sakuhara

30 received a JSPS KAKENHI Grant-in-Aid for Scientific Research (C), and a grant from AMED, unrelated

31 to this study. Takeshi Soyama received a JSPS KAKENHI Grant-in-Aid for Scientific Research (C),

32 unrelated to this study. Norio Katoh received a JSPS KAKENHI Grant-in-Aid for Scientific Research

33 (C), unrelated to this study. Naoki Miyamoto received a JSPS KAKENHI Grant-in-Aid for Scientific 
34 Research (B), and a JSPS KAKENHI Grant-in-Aid for Challenging Research (Exploratory), unrelated to

35 this study. Shinichi Shimizu received grants from AMED, Hitachi Co., Ltd., a JSPS KAKENHI Grant-in-

36 Aid for Scientific Research (B), unrelated to this study. Hiroki Shirato received grants from AMED,

37 Hitachi Co., Ltd., and Shimadzu Co., Ltd., unrelated to this study. Hiroki Shirato has a patent for a

38 moving body pursuit irradiating device and a positioning method using this device, with royalties paid to

39 Hokkaido University. Kohsuke Kudo received a JSPS KAKENHI Grant-in-Aid for Scientific Research

40 (B), personal fees from GE Healthcare, Hitachi Medical Systems, Philips Medical Systems, Toshiba

41 Medical Systems, Siemens Medical Systems, Cannon Marketing Japan, Bayer Healthcare, Daiichi-

42 Sankyo Healthcare, and grants from AMED, unrelated to this study.

43 IRB approval: This study has obtained IRB approval from Hokkaido University Hospital and the need

44 for informed consent was waived.

45

46 


\section{Percutaneous insertion of hepatic fiducial true-spherical markers for}

Purpose: This study evaluated the success rate and complications of percutaneous

implantation of hepatic fiducial true-spherical gold markers for real-time adaptive radiotherapy (RAR), which constitutes real-time image-guided radiotherapy with gating.

Materials and Methods: We retrospectively evaluated 100 patients who underwent 116

percutaneous intrahepatic implantations of 2-mm-diameter, spherical, gold fiducial

markers before RAR from 1999 to 2016, using Seldinger's method. We defined technical

success as marker placement at intended liver parenchyma, without mispositioning, and

clinical success as successful tracking of the gold marker and completion of planned

RAR. Complications related to marker placement were assessed.

Results: The technical success rate for true-spherical gold marker implantation was 
were encountered.

\section{Introduction}

71 radiotherapy, has been reported as an effective alternative to radical surgery for hepatic

72 malignant tumors [1]. Because its success greatly depends on the accuracy of daily set-

73 up of the patient, as well as the management of respiratory tumor motion, image-guided

74 radiation therapy (IGRT) using imaging equipment in the treatment room is expected to

75 further improve the clinical results. Daily set-up of the patient has been shown to be

76 improved by IGRT using on-line X-ray cone beam computed tomography (CBCT),

77 deformable registration software, four-dimensional reconstruction of CBCT, and on-line magnetic resonance imaging (MRI) $[2,3,4]$. For the management of respiratory tumor 
motion during irradiation, real-time adaptive radiotherapy (RAR) is expected to reduce

uncertainty [5]. RAR is defined as radiotherapy that, throughout therapeutic irradiation,

81 monitors patient anatomy or physiology; based upon that information, RAR allows

autonomous adjustments of treatment parameters during therapeutic irradiation without

operator intervention [5]. In an RAR system in the clinic, internal motion within tumors

can be automatically tracked 30 times per second by 2 sets of fluoroscopy images with

real-time pattern recognition of implanted fiducial markers; the therapeutic beam is

controlled to irradiate/not to irradiate the patient with 0.05 -second intervals for gating

$87[6,7]$. Recently, an advanced proton RAR system has also been developed for use in treatment of large hepatic tumors [8].

As a surrogate marker for hepatic tumors, the fiducial marker is required to be

90 implanted as close as possible to the tumor [9]. There have been several reports

91 regarding the transarterial hepatic implantation of an embolization coil [10] and

92 percutaneous transhepatic implantation of a gold marker [11-17]. The former is more

93 time-consuming, while the latter is technically simpler and more often used. In both

94 techniques, non-spherical markers with a size of approximately $1 \times 3 \mathrm{~mm}$ have 
96 are used for automatic precise calculation of the gravity center of the marker in RAR for

97 hepatic malignant tumors [18,19]. However, only a few studies with a small number of

98 patients have assessed the safety and efficacy of percutaneous transhepatic marker

99 implantation of true-spherical markers $[18,19]$ and the complications and migration of

100 true-spherical markers remain unclear.

101 This is the first study in which the efficacy and safety of percutaneous

102 transhepatic implantation of true-spherical markers for RAR were evaluated with a

103 sufficient number of patients for statistical analysis.

104 Materials and methods

105 Patients Institutional review board approval was obtained for this retrospective review,

107 and a Health Insurance Portability and Accountability Act waiver was granted. Medical records of 148 patients who underwent percutaneous implantation of

109 fiducial gold markers into the liver as preparation for RAR at our hospital from January

1101999 to June 2016 were retrospectively reviewed. Of these patients, 48 were excluded

111 for the following reasons: i) fluoroscopic images or computed tomography (CT) images

112 were not acquired immediately post-procedure $(n=21)$, ii) no detailed procedure report 
113 was available $(\mathrm{n}=22)$, iii) radiation therapy was not performed in our hospital $(\mathrm{n}=1)$,

114 and iv) radiation therapy was cancelled due to the patients' poor general condition $(\mathrm{n}=$

115 4). Consequently, 100 patients were evaluated in this study. The patients included 73 men and 27 women (median age, 66 years; range, 38-

11789 years). All patients, except 1 , had hepatobiliary cancers: 81 hepatocellular

118 carcinomas, 15 metastatic hepatic tumors, and 3 intrahepatic cholangiocarcinoma. The

119 remaining patient had extrahepatic lymph node metastasis of gastric cancer, close to the

120 liver. The indication of RAR for hepato-biliary cancers was as follows: i) Karnofsky

122 performance status $\geq 70 \%$, ii) Child-Pugh classification A or B, iii) tumor detectable on

$123 \mathrm{CT}$ and/or magnetic resonance imaging (MRI), iv) 3 or fewer lesions without

124 extrahepatic metastases, v) ineligible for, or refusal of any other locoregional therapy,

125 e.g., surgery, radiofrequency ablation, and transcatheter arterial chemoembolization.

126 The type of radiation (X-ray or protons) used depended on tumor size, liver function,

127 patients' health, and insurance coverage. In the patient with lymph node metastasis of

128 gastric cancer, no hepatic metastasis was found, but RAR was indicated because this patient had sole lymph node metastasis in the para-aortic space. The liver was selected 
130 as the site for implantation of the fiducial marker due to its proximity to the target

131 lymph node.

133 (115 tumors in total). The median diameter of the target tumor was $24 \mathrm{~mm}$ (range: 7-

$134154 \mathrm{~mm})$. Tumor locations are summarized in Table 1.

136 planned, because radiation oncologists estimated that 1, 2, or 3 markers were needed for

137 RAR of 103 tumors in 95 patients, 9 tumors in 4 patients, and 3 tumors in 1 patient,

138 respectively. Only 1 marker was placed for 2 or more tumors when they were in close 139 proximity.

\section{RAR Equipment}

141 The RAR systems employing implanted fiducial markers in our hospital are the

142 real-time tumor-tracking radiotherapy (RTRT) system (Varian Medical Systems,

143 Mitsubishi Electric, Tokyo, Japan) and real-time image-gated proton beam therapy

144 (RGPT) system (Hitachi, Tokyo, Japan). They allow delivery of X-ray in RTRT and

145 proton beam in RGPT when the implanted fiducial marker is within the gating window, 
146 facilitating delivery of a precise radiation dose to the tumor while sparing the adjacent

147 normal tissues.

\section{Implantation procedure}

All procedures were performed in the angiographic suite with a combined CT

150 and angiography system (Artis zee TA, SOMATOM Definition AS64, Siemens,

151 Munich, Germany). We aimed to implant the gold marker in the hepatic parenchyma

152 within approximately $\leq 5$ or $10 \mathrm{~cm}$ from the center of the tumor for RTRT or RGPT,

153 respectively. When the tumor diameters exceeded $10 \mathrm{~cm}$ in RTRT, we implanted the

154 gold marker as close to the tumor as possible. The location was chosen by consensus of

155 the interventional radiologist(s) and radiation oncologist(s) prior to the procedure.

156 All marker implantations were performed, using Seldinger's method, by 7

157 board-certified interventional radiologists (with 5-20 years of experience in

158 interventional radiology). A safe puncture tract, avoiding major vessels, was determined

159 by liver ultrasonography; then, the liver parenchyma was percutaneously punctured

160 under US guidance (Figure 1a), using an 18-gauge needle (Needle for Ultrasonically

161 Guided Puncture, Create Medic, Kanagawa, Japan). Intra-procedural CT was performed 
162 if the inserted needle tip was obscure, or the needle direction was uncertain under US-

163 or fluoroscopic-guidance alone.

164 A guidewire (0.035 Amplatz Ultra Stiff Wire Guide, Cook, Bloomington,

165 Indiana, USA, or 0.035 Fixed Core Wire 3-mm J Guide wire, Argon Medical Devices,

166 Dallas, Texas, USA) was inserted into the liver parenchyma through the needle (Figure

$167 \mathrm{1b}$ ), followed by the introduction of a 2.55 -mm-diameter sheath introducer (Introducer

168 Set, Medikit, Tokyo, Japan) (Figure 1c) designed for marker implantation (Figure 1d),

169 under X-ray fluoroscopic guidance. After removal of the guidewire and inner tube, the

170 absence of blood or bile juice reflux was confirmed through a 3-way stopcock of the

171 sheath with a 10-mL syringe, to ensure that the gold marker was not placed in the

172 vessels or bile duct. If reflux was observed, the sheath was pulled back or a gelatin

173 sponge torpedo (Spongel, Astellas Pharma, Tokyo, Japan) was introduced into the tip of

174 the sheath and the presence of reflux again checked. When reflux was absent, a 2-mm-

175 diameter, spherical, pure gold marker (iGold, Medikit, Tokyo, Japan) (Figure 1e) was

176 pushed into the liver parenchyma through the sheath, by means of the pusher (Figure

177 1f). The sheath was then extracted while packing the puncture tract with gelatine sponge

178 torpedoes. After removing the sheath, the site of the implanted marker was confirmed 
180 migration of the marker was of concern. If migration was confirmed on US,

181 fluoroscopy, or CT, the complete procedure was repeated with an additional marker; such implantation was counted as an additional procedure in this study.

184 the surface of the liver, and the patient was placed on complete bed rest for 2 hours. On

185 the day after the implantation procedure, contrast-enhanced planning CT (Figure 1h)

186 was performed to evaluate the marker position and to monitor for complications, such

187 as intra-abdominal hemorrhage. One day before the start of radiotherapy, diagnostic CT

188 was performed to determine whether the marker was dislocated from its planned

189 position in RGPT. This was followed by abdominal fluoroscopic imaging and/or set-up

190 imaging at each fraction on RAR to check the marker position.

\section{Evaluation of efficacy and safety}

192 Technical and clinical success rates were evaluated. Technical success was

193 defined as completion of gold marker implantation into the hepatic parenchyma. When

194 migration occurred, the implantation procedure was defined as a technical failure.

195 "Migration" was defined as migration of the gold marker from the initial site of 
196 implantation in the hepatic parenchyma to other sites, such as the extra-hepatic region

197 or intrahepatic vessels (portal vein, hepatic vein, or bile duct). Migration was

198 categorized as intraprocedural migration when it was recognized during the

199 implantation procedure, and as delayed migration when it was recognized after the

200 procedure. Delayed migration was detected during the daily set-up of patients for

201 radiotherapy. After alignment of the patient on the treatment couch, using a bony

202 structure for reference in orthogonal X-ray imaging, the 3D position of the marker and

203 its trajectory relative to the bony structure were checked using 2 sets of orthogonal

204 fluoroscopy imaging. If the dislocation of the marker from its planned position

205 exceeded $2 \mathrm{~mm}$, even after several manual maneuvers, such as re-arranging the patient's

206 body or instructing the patient to stand upright for a period of time, the patient

207 underwent additional CT scanning to check for delayed migration.

208 Technical marker placement success was evaluated by a board-certified

209 interventional radiologist, based on post-procedural CT or planning CT. Migration was

210 checked during and after the implantation procedure until all RAR sessions had been

211 completed. The technical success rate per procedure was calculated. The causes of

212 migration were also assessed according to medical records. 
214 implanted gold marker and planned RAR. When the implanted marker could not be

215 utilized for tracking, the marker implantation was defined as a clinical failure.

216 Complications after percutaneous marker implantation procedure were recorded

217 according to the information obtained from the referring physician and medical records.

218 These complications were evaluated based on the Common Terminology Criteria for

219 Adverse Events v4.0 (CTCAE). Minor complications were defined as CTCAE grades 1

220 and 2, and major complications were defined as grade 3 or higher.

$221 \quad$ Results

\section{Technical Success}

Of the 106 planned fiducial marker implantations, $98(92.5 \%) 1^{\text {st }}$ implantations

224 were successful, without migration (Figure 2), while 8 migrated. Of these 98

225 implantations, 2 patients underwent successful $2^{\text {nd }}$ marker implantations during the

226 same session because mispositioning of the $1^{\text {st }}$ implanted marker was a concern for the

227 attending physician. Four markers were implanted in the extra-hepatic region and

another 4 markers migrated from the initial implantation site in the hepatic parenchyma, 
230 migrations, 6 were confirmed intraprocedurally, and the 2 migrations into the bile duct

231 were delayed.

In the 6 intraprocedural migrations, successful $2^{\text {nd }}$ marker implantation was

233 performed in 5 cases. In the remaining patient, the marker was confirmed to be located

234 in the intrahepatic portal vein at the periphery of hepatic segment 3 ; no additional

235 marker was implanted, because the marker position (in a small portal vein) was

236 expected to be stable.

237 Delayed migration of 2 markers into the bile duct were diagnosed 1 and 14 days

238 post-procedurally, although the operator initially considered the marker implantations

239 successful. A $2^{\text {nd }}$ marker implantation was successfully performed in 1 patient. The

240 other patient underwent $2^{\text {nd }}$ marker implantation; however, the marker migrated into the

241 hepatic vein. A $3^{\text {rd }}$ marker was then successfully implanted during the same session.

242 In summary, 106 procedures were initially planned; the initial success rate was

$24392.5 \%(98 / 106) ; 2^{\text {nd }}$ and $3^{\text {rd }}$ procedures were planned for 9 and 1 markers, respectively,

244 and 116 procedures were conducted in total. Of these, 107 markers were successfully

245 implanted at the hepatic parenchyma without migration, in 1-3 implantation

246 procedure(s). Thus, the technical success rate per procedure was estimated as $92.2 \%$. 
247 Among the 116 markers, 9 implantations were technical failures (due to migration).

248 Nine of the $116(7.8 \%)$ implanted markers migrated; 8 and 1 migrations occurred at the

$2491^{\text {st }}$ and $2^{\text {nd }}$ implantation procedures, respectively. Four markers were implanted into the

250 extra-hepatic region because of the unexpected dislocation of the sheath introducer, due

251 to respiratory movement during the procedure. Migration into the portal vein and

252 hepatic vein occurred when the sheath was extracted during packing of the puncture

253 tract. For the remaining 3 markers, the reasons for migration were unknown, due to lack

254 of detailed medical records.

255 Clinical Success

256 Among 115 tumors in 100 patients, all implanted markers were successfully

257 tracked by the RTRT or RGPT systems and the planned treatment was completed.

258 Accordingly, the clinical success rate was $100 \%(115 / 115)$ per tumor and $100 \%$

$259(100 / 100)$ per patient.

260 Complications/Adverse Events

261 Among the 100 patients, abdominal pain after the procedure occurred in 16

262 patients (grade 1 in 6 patients, grade 2 in 10 patients), mild fever occurred in 7 (all 
263 grade 1), hemorrhage occurred in 7 (all grade 1), and pneumothorax occurred in 1

264 patient (grade 1). All adverse events were treated conservatively. Blood transfusion and

265 chest tube insertion were not necessary. No major complications occurred. No

266 complications associated with migrated markers were observed up to the end of RAR,

267 although the migrated markers were not retrieved.

\section{Discussion}

Percutaneous implantation of fiducial marker(s) is simple, can be easy and

rapidly performed, and is widely accepted as a routine practice for improving accuracy

271

$[13,16]$. Non-spherical fiducial gold markers (Figure 3 ) can be simply implanted by

$27497.3 \%-100 \%$ has been reported for percutaneous implantation of hepatic non-spherical

275 fiducial markers (Figure 3) [11, 14, 15, 20]. In contrast, implanting a 2-mm-diameter,

276 true-spherical gold marker requires insertion of a sheath (2.55-mm-diameter)

277 specifically designed for marker implantation after insertion of the needle into the liver

278 [18]. This is the first study to demonstrate a high technical success rate for implantation

279 of true-spherical fiducial markers $(92.2 \%)$ with a relatively large number of patients. 
281 that reported previously for non-spherical fiducial markers $(0.9 \%-1.5 \%)[12,17]$. Four

282 of 9 migrating markers in our study involved dislocation of the sheath introducer due to respiratory organ movement. Accordingly, use of a sheath introducer could lower the

284 technical success rate. Avoiding dislocation may require insertion of the sheath

285 introducer as deeply as possible, with a long intrahepatic tract, to stabilize it against

286 respiratory motion. The remaining 5 markers migrated into intrahepatic vessels. The

287 proximity of the tumor to a major hepatic vein could cause migration [21]. Additionally,

288 the shape of true-spherical markers may facilitate migration. We observed 2 migrations

289 when the sheath was extracted while packing the puncture tract with gelatine sponge

290 torpedoes. If a marker migrates into the right atrium, it should be retrieved because it

291 can cause arrhythmia [21] or systemic embolization in patients with right-to-left cardiac

292 shunting [22]. However, we did not experience any migration-associated complications.

293 Chan et al. compared visibility of various implantable non-spherical markers

294 [23] and concluded that, for IGRT, markers with at least a $0.75-\mathrm{mm}$ diameter should be

295 selected for hybrid kilovoltage $(\mathrm{kV}) / \mathrm{mV}$ imaging (the worst condition for marker

296 visualization). Because non-spherical markers are usually tiny cylinders with a small 
297 short-axis diameter $(0.75-1.1 \mathrm{~mm})$, they may be poorly visible on X-ray images,

298 especially when they are projected in the short-axis plane in patients who are obese or

299 muscular and may not be useful for tracking of markers in RAR, such as in RTRT and

300 RGPT.

301 A 2-mm-diameter, true-spherical, pure gold marker has much better visibility

302 than non-spherical markers due to its larger diameter [11, 13-16]. Therefore, the

303 technical success rate (per procedure) and complication rate in this study, which was

304 slightly worse than those reported previously for non-spherical markers

$305[11,12,14,15,17]$, seems acceptable, as this true-spherical marker theoretically provides

306 the best likelihood of usability for tumor tracking. The accuracy in calculating the 3D

307 co-ordinates of the gravity center of a marker is logically better with a spherical marker

308 than with a non-spherical marker [24].

Implantation of 3 or more markers is usually required per patient [13]. Although

310 multiple fiducial markers can be beneficial for localizing the target, they produce more

311 unfavorable artifacts in CT scans, which may affect the target volume delineation and

312 dose calculation. We implanted 116 true-spherical markers in 100 patients with 115

313 lesions (1.2 and 1.0 markers per patient and per lesion, respectively), while Jarraya et al. 
314 implanted 1444 non-spherical markers in 328 patients with 424 hepatic lesions (4.4 and

3153.4 markers per patient and per hepatic lesion, respectively) [17]. Making multiple liver

316 punctures for implanting multiple markers are potentially time-consuming and more

317 invasive. Therefore, our technical success rate was reasonable. If markers are placed

318 within $2 \mathrm{~mm}$ from their planned positions in RTRT and RGPT, use of multiple markers

319 has no added benefit. However, if irradiation of the tumor involves dynamic direction

320 changes of the therapeutic beam, multiple markers will be required to adjust for rotation

321 and distortion of the tumor during respiration.

$323[12,13]$, such as major bleeding or sepsis, at a reported rate of $1.6 \%$ after marker

324 placement in the abdomen or pelvis [12]. A lower major complication rate of $0.6 \%$

325 (pneumothorax and biloma in 1 patient each) has been reported for marker placement in

326 the liver [17]. However, we encountered only minor complications: 7 mild fevers, 7

327 hemorrhages, and 1 pneumothorax, which were all conservatively treated. Therefore,

328 we consider our technique to be safe.

In future, the development of spherical or semi-spherical gold markers that can be implanted by a simple needle insertion technique, such as that used for non-spherical 
331 markers, while preserving visibility similar to that of a 2-mm-diameter, true-spherical,

332 pure gold marker, may yield a higher success rate with less invasiveness, and a lower

333 complication rate.

This study had several limitations. First, it was retrospective in nature. Second,

approximately one-third of patients who underwent marker implantation were excluded

due to lack of imaging data and/or procedure reports. Third, implantation procedure

337 details might have changed between the initial and later phases, because different

338 physicians were involved. These factors could affect estimation of the success and

339 complication rates. For the assessment of delayed migration, diagnostic CT was

340 performed to check whether the marker was dislocated from its planned position in

341 RGPT, 1 day before the start of RGPT, but not in RTRT, during this period. We now

342 recommend CT evaluation for delayed migration 1 day before either RGPT or RTRT

$343[25]$.

There are high expectations regarding RAR without fiducial markers, such as

345 on-line MRI in the treatment room, for the treatment of liver tumors. However, only 2-

346 dimensional MRI movies have been used thus far [26]. In addition, automatic real-time 
348 gating of irradiation [26]. Therefore, it remains important to implant fiducial markers

349 into the liver in real-world RAR.

350 In conclusion, percutaneous transhepatic implantation of true-spherical gold

351 markers facilitate RAR; it is technically feasible and can be conducted with a

352 reasonably high success rate and low complication rate by interventional radiologists.

353 Acknowledgments: The authors wish to acknowledge the kind support of Osamu Sugita,

354 Takeshi Uchida, Yoriko Ida, Fujio Inage, and Kenichiro Maeda.

355 
357 [1] Su TS, Liang P, Liang J, et al. Long-term survival analysis of stereotactic ablative radiotherapy versus liver resection for small hepatocellular carcinoma. Int J Radiat Oncol Biol Phys. 2017;98:639-646.

360 [2] Zhang Y, Folkert MR, Li B, et al. 4D liver tumor localization using cone-beam projections and a biomechanical model. Radiother Oncol. 2019;133:183-192.

362 [3] Wang J, Gu X. Simultaneous motion estimation and image reconstruction (SMEIR) for 4D cone-beam CT. Med Phys. 2013;40:101912.

364 [4] van de Lindt TN, Fast MF, van Kranen SR, et al. MRI-guided mid-position liver radiotherapy: Validation of image processing and registration steps. Radiother Oncol. 2019;138:132-140.

367 [5] IEC TR 62926-Ed.1.0:2019-5: Medical electrical system - Guidelines for safe integration and operation of adaptive external-beam radiotherapy systems for real-time adaptive radiotherapy.

370 [6] Taguchi H, Sakuhara Y, Hige S, et al. Intercepting radiotherapy using a real-time tumor-tracking radiotherapy system for highly selected patients with 

Biol Phys. 2007; 69:376-380.

374 [7] Katoh N, Soda I, Tamamura H, et al. Clinical outcomes of stage I and IIA non-small cell lung cancer patients treated with stereotactic body radiotherapy using a realtime tumor-tracking radiotherapy system. Radiat Oncol. 2017;12:3.

[8] Shimizu S, Miyamoto N, Matsuura T, et al. A proton beam therapy system dedicated to spot-scanning increases accuracy with moving tumors by real-time imaging and gating and reduces equipment size. PLoS One. 2014;9:e94971.

[9] Park JC, Park SH, Kim JH, et al. Liver motion during cone beam computed

[10] Ohta K, Shimohira M, Sasaki S, et al. Transarterial fiducial marker placement for tomography guided stereotactic body radiation therapy. Med Phys.

2012;39:6431-6442.

[10] Ohta K, Shimohira M, Sasaki S, et al. Transarterial fiducial markerplacement for image-guided proton therapy for malignant liver tumors. Cardiovasc Intervent Radiol. 2015;38:1288-1293. 2009;20:235-239. 
389 [12] Brook OR, Gourtsoyianni S, Mendiratta-Lala M, et al. Safety profile and technical success of imaging-guided percutaneous fiducial seed placement with and without core biopsy in the abdomen and pelvis. AJR Am J Roentgenol. 2012;198:466-470.

[13] Kim JH, Hong SS, Kim JH, et al. Safety and efficacy of ultrasound-guided fiducial marker implantation for CyberKnife radiation therapy. Korean J Radiol. 2012;13:307-313. intrahepatic fiducial markers for proton beam therapy of liver lesions: 2015;204:W207-213.

400 [15] Trumm CG, Haussler SM, Muacevic A, et al. CT fluoroscopy-guided percutaneous fiducial marker placement for CyberKnife stereotactic radiosurgery: technical results and complications in 222 consecutive procedures. J Vasc Interv Radiol. 
404 [16] Ohta K, Shimohira M, Murai T, et al. Percutaneous fiducial marker placement prior to stereotactic body radiotherapy for malignant liver tumors: an initial experience. J Radiat Res. 2016;57:174-177.

407 [17] Jarraya H, Chalayer C, Tresch E, et al. Novel technique for hepatic fiducial marker placement for stereotactic body radiation therapy. Int J Radiat Oncol Biol Phys.

409 2014;90:119-125.

410 [18] Kitamura K, Shirato H, Shimizu S, et al. Registration accuracy and possible migration of internal fiducial gold marker implanted in prostate and liver treated with real-time tumor-tracking radiation therapy (RTRT). Radiother Oncol. 2002;62:275-281.

414 [19] Shirato H, Harada T, Harabayashi T, et al. Feasibility of insertion/implantation of 2.0-mm-diameter gold internal fiducial markers for precise setup and real-time tumor tracking in radiotherapy. Int J Radiat Oncol Biol Phys. 2003;56:240-247.

417 [20] Park SH, Won HJ, Kim SY, et al. Efficacy and safety of ultrasound-guided implantation of fiducial markers in the liver for stereotactic body radiation therapy. PLoS One. 2017;12:e0179676. 
420 [21] Hennessey H, Valenti D, Cabrera T, et al. Cardiac embolization of an implanted fiducial marker for hepatic stereotactic body radiotherapy: a case report. J Med Case Rep. 2009;3:140.

[22] Zhu AX, Wallner KE, Frivold GP, et al. Prostate brachytherapy seed migration to the right coronary artery associated with an acute myocardial infarction. Brachytherapy. 2006;5:262-265.

[23] Chan MF, Cohen GN, Deasy JO. Qualitative evaluation of fiducial markers for radiotherapy imaging. Technol Cancer Res Treat. 2015;14:298-304.

[24] Shirato H, Shimizu S, Kunieda T, et al. Physical aspects of a real-time tumortracking system for gated radiotherapy. Int J Radiat Oncol Biol Phys. needed? Radiother Oncol. 2016;121:75-78.

434 [26] Bertholet J, Knopf AC, Eiben B, et al. Real-time intrafraction motion monitoring 435 in external beam radiotherapy. Phys Med Biol 2019. doi:10.1088/13616560/ab2ba8. 


\section{Figure Legends}

438 Figure 1 Procedure of percutaneous transhepatic implantation of true-spherical gold marker. (a)

439 Safe puncture tract (white arrows) is decided by scanning of the liver with ultrasonography

440 (US). (b) Fluoroscopic image shows insertion of the 0.035 -inch guidewire (black arrow head)

441 through an 18-gauge needle (black arrows) percutaneously punctured with US guidance. (c)

442 Digital photograph shows a percutaneously placed $2.55-\mathrm{mm}$ diameter sheath introducer (black

443 arrow) after removal of the guidewire and inner tube. (d) Digital photograph shows a 2.55-mm

444 diameter sheath introducer (Introducer Set, Medikit, Tokyo, Japan) (white arrow) designed for

445 marker implantation, and the pusher (white arrowhead). (e) Digital photograph shows a 2-mm

446 diameter spherical pure gold marker (iGold, Medikit, Tokyo, Japan) close to the tip of the

447 sheath introducer, which has a radiopaque marker (white arrow). (f) A 2-mm-diameter,

448 spherical pure gold marker is pushed into the liver parenchyma through the sheath (black arrow)

449 by means of the pusher (black arrowhead). (g) Fluoroscopic image shows the true-spherical

450 fiducial marker implanted in the liver parenchyma (white arrowhead). (h) Contrast enhanced-

451 computed tomography for planning of real-time adaptive radiotherapy (RAR), obtained 1 day

452 after marker implantation to evaluate the marker position, shows true-spherical fiducial marker

453 (black arrow) in the liver parenchyma. 
454 Figure 2 Details of marker malposition.

455 Figure 3 Various non-spherical fiducial markers for image-guided radiation therapy (IGRT).

456 Figure 4 A representative non-spherical fiducial marker and its implantation system.

457 (a) A 1.1-mm-diameter, 10-mm-long non-spherical fiducial marker (Visicoil, IBA Dosimetry,

458 Schwarzenbruck, Germany) (single asterisk) is composed of a kit with a 17-gauge needle (white

459 arrow), inner stylet (white arrowhead), and the stopper (double asterisk). (b) A marker is set at

460 the needle tip and the marker kit is assembled for the percutaneous implantation procedure. (c)

461 The stopper is removed when the tip of the needle reaches the target site. Next, the needle is

462 pulled over the inner stylet, holding the inner stylet in place. (d) The marker set at the needle tip

463 is placed in the target site by pushing the inner stylet.

464 
Table 1. Tumor locations

\begin{tabular}{cc}
\hline Location of target tumors & Number of tumors \\
\hline S1 & 9 \\
S2 3 & 5 \\
S4 & 11 \\
S5 & 14 \\
S6 & 9 \\
S7 & 6 \\
S8 & 5 \\
Right lobe ( $>2$ segments $)$ & 24 \\
Left lobe (>2 segments) & 15 \\
Portal vein tumor thrombus & 7 \\
Hepatic vein tumor thrombus & 8 \\
Lymph node metastasis & 1 \\
\hline
\end{tabular}

$\mathrm{S} 1$ = caudate lobe; $\mathrm{S} 2=$ dorsolateral segment of left lobe; $\mathrm{S} 3=$ ventrolateral segment of left lobe; $\mathrm{S} 4=$ medial segment of left lobel S5 = anteroinferior segment of right lobe; S6 = posteroinferior segment of right lobe; S7 = posterosuperior segment of right lobe; S8 = anterosuperior segment of right lobe. 
Figure 1
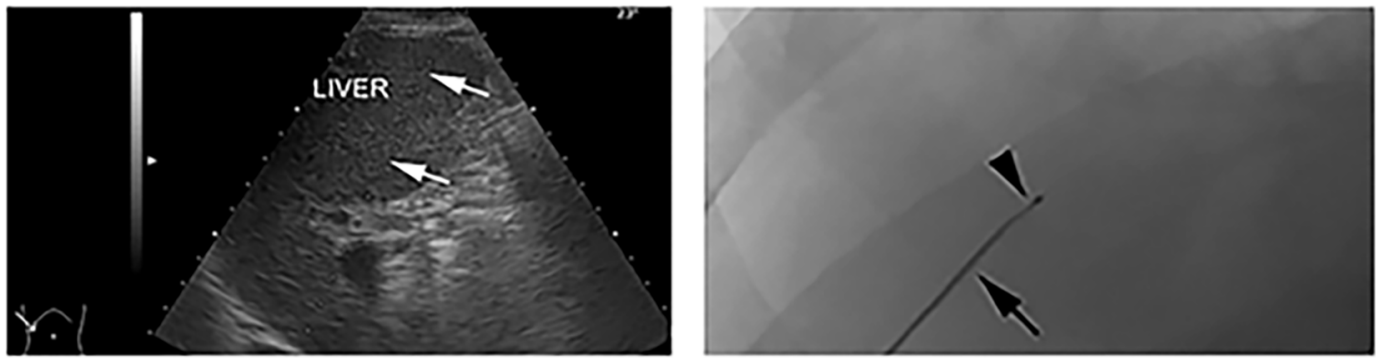

a.

b.
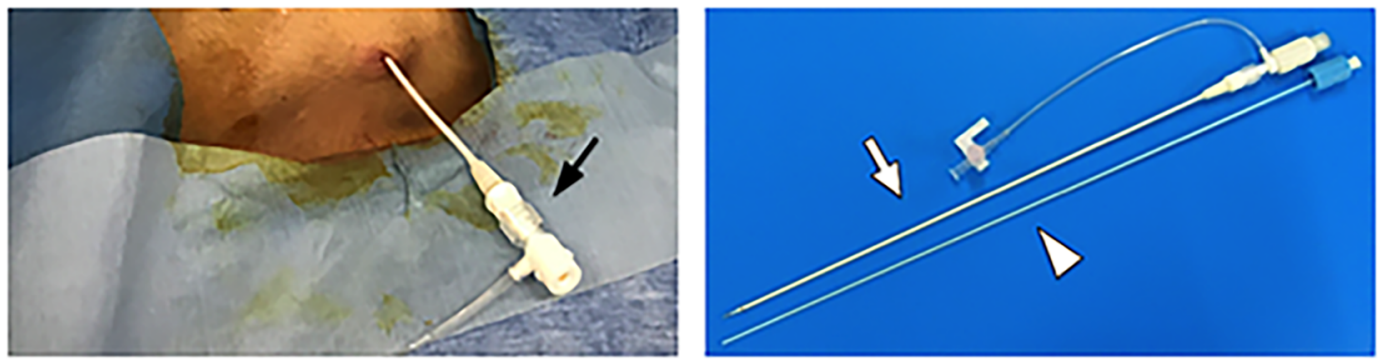

c.

d.
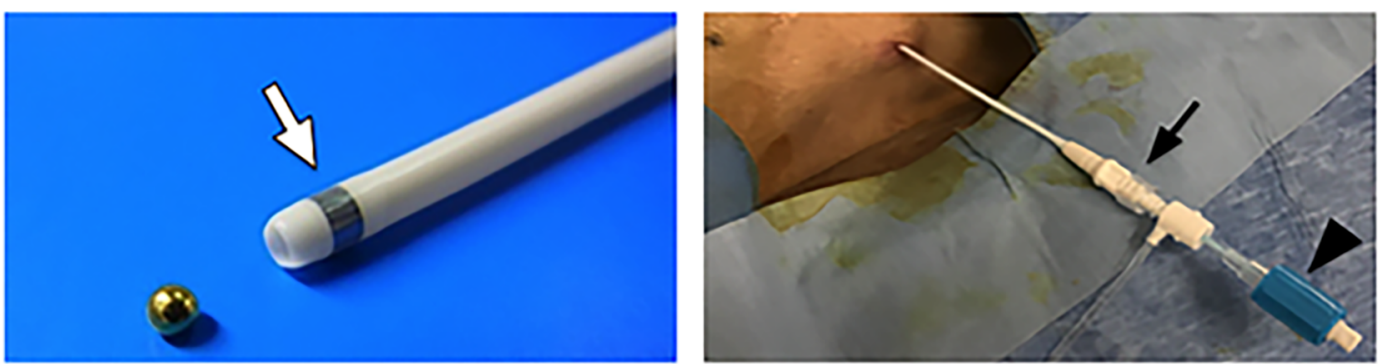

e.

f.
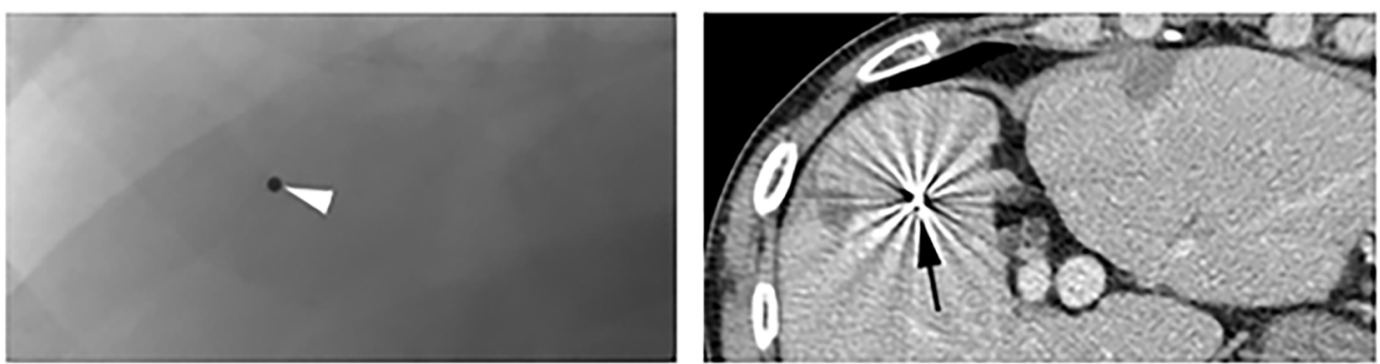

g.

h. 
Figure 2

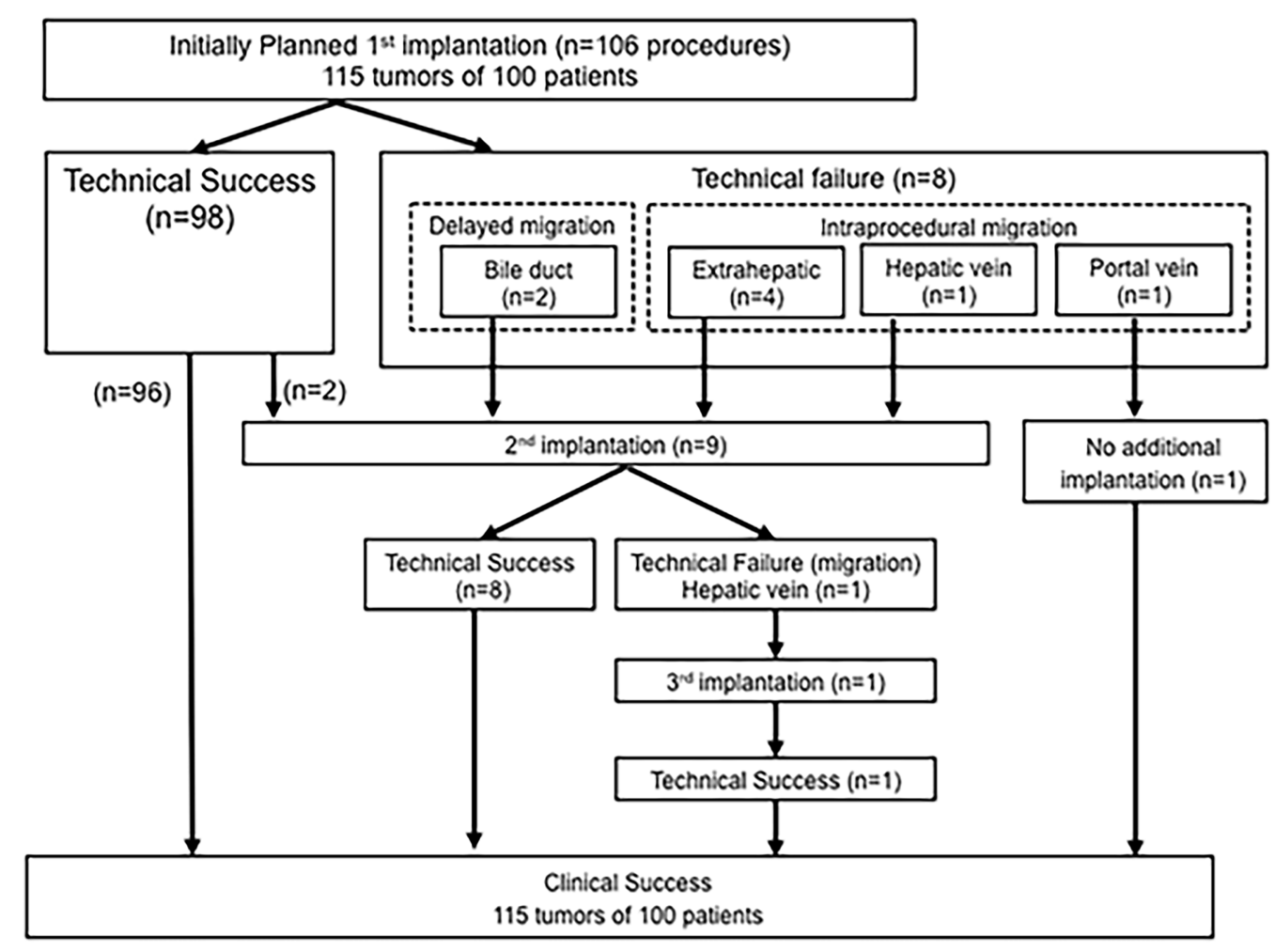


Figure 3

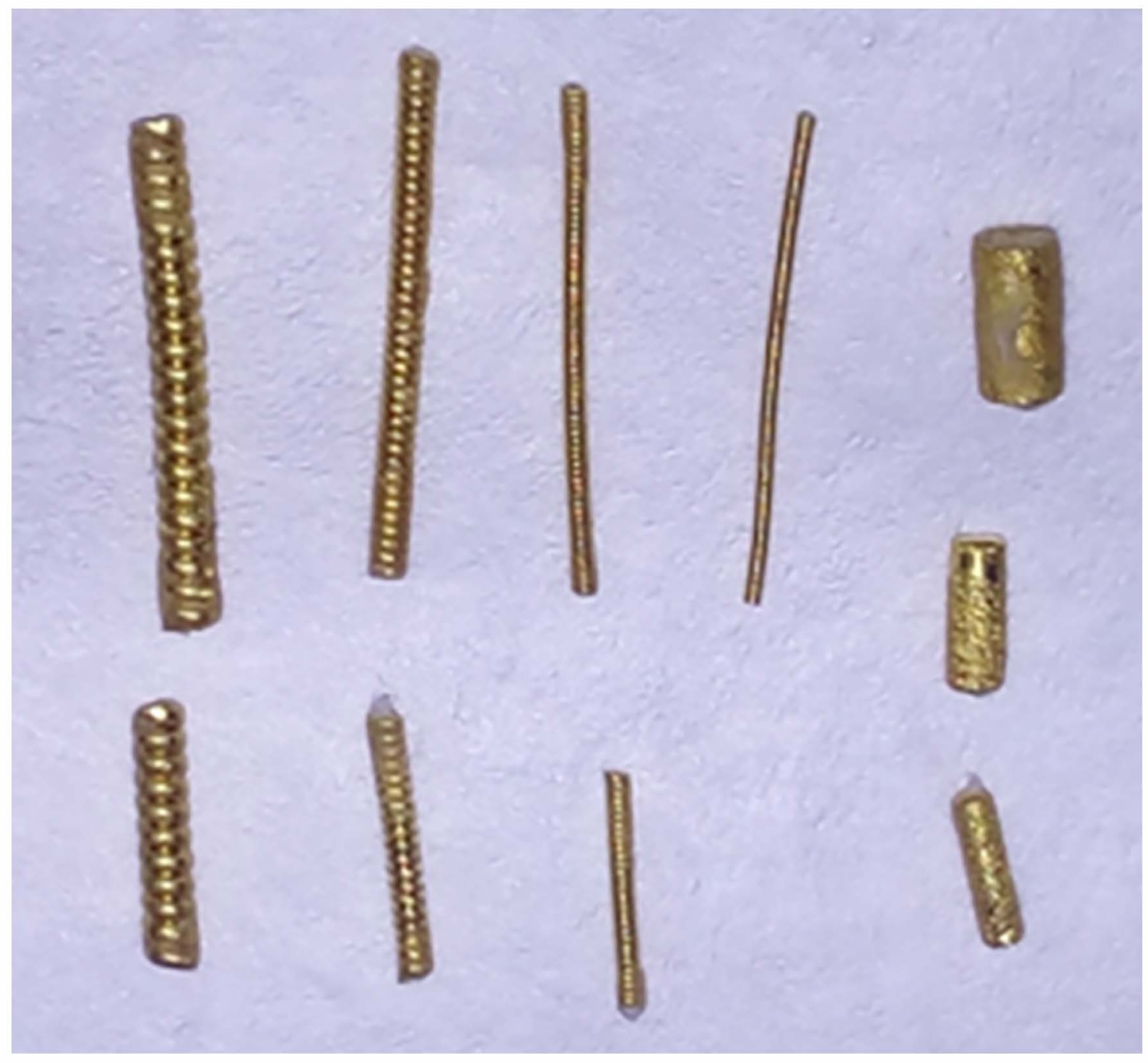


Figure 4

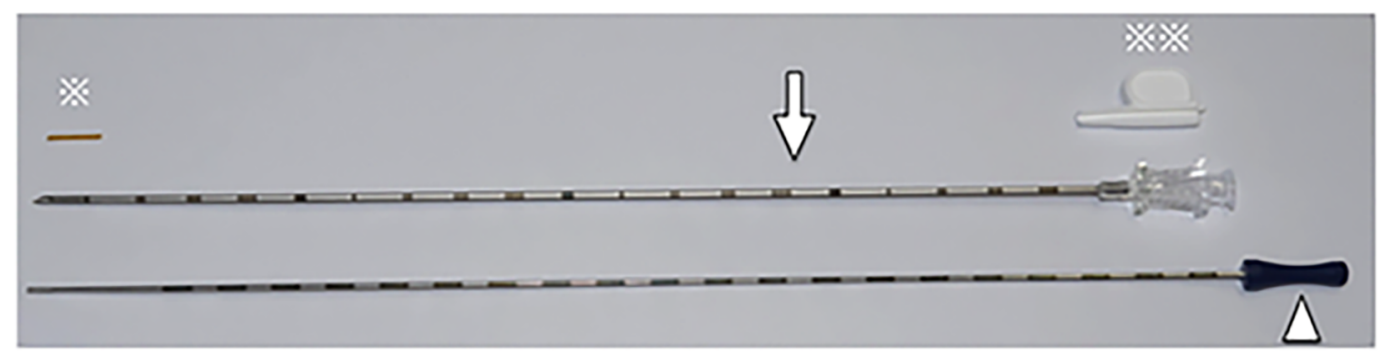

a.

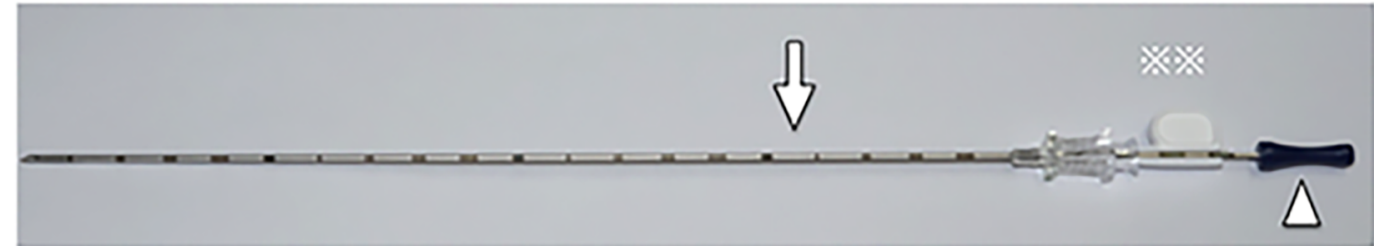

b.

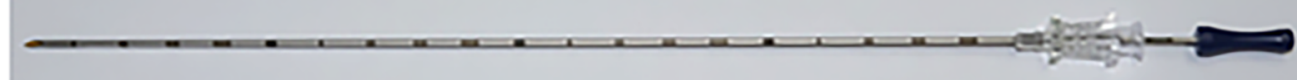

c.

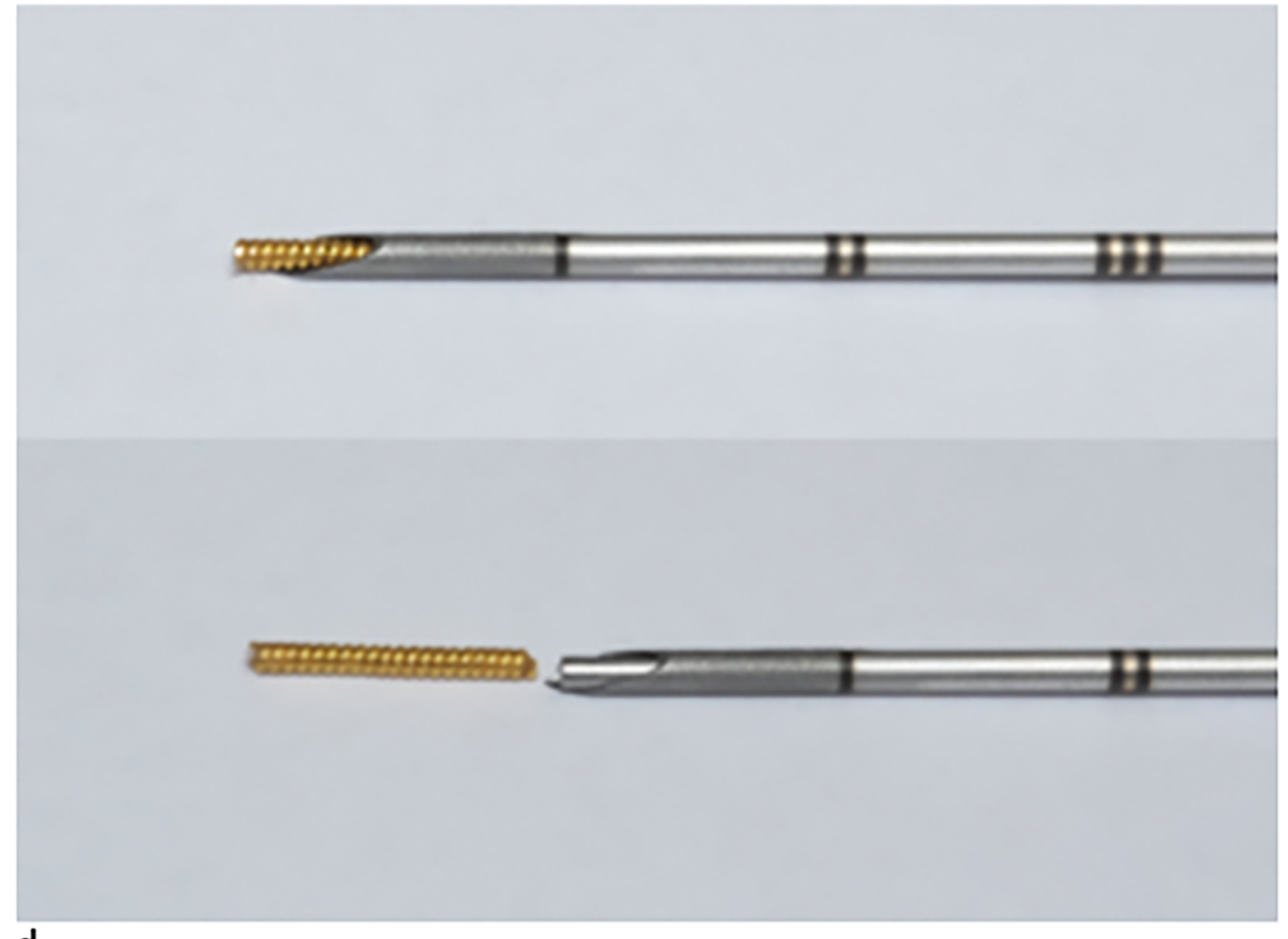

d. 